Bull. Austral. Math. Soc.

$15 \mathrm{~A} 18,05 \mathrm{c} 50$

VOL. 53 (1996) [189-196]

\title{
SIGN PATTERN MATRICES THAT ALLOW A NILPOTENT MATRIX
}

\section{LINA YEH}

We characterise some star sign pattern matrices and linear tree sign pattern matrices that allow a nilpotent matrix.

\section{INTRODUCTION}

A sign pattern matrix $A$ is an $n \times n$ matrix whose entries consist of the symbols ,+- and 0 . Let $Q(A)$ denote the set of all $n \times n$ real matrices that have the same sign pattern as $A$. For a property $P$, a sign pattern matrix $A$ is said to require (respectively, allow) $P$ if every (respectively, there exists a) matrix in $Q(A)$ (respectively, that) has property $P$. In [3], Eschenbach and Johnson characterised sign pattern matrices that require some eigenvalue properties, and they asked [2] several questions about the sign pattern matrices that require or allow a distribution of eigenvalues. Typically they ask for a characterisation of sign pattern matrices that allow a nilpotent matrix. In this paper, we examine this question for star sign pattern matrices and linear tree sign pattern matrices of order less than 8. First we review some notation and definitions that are needed to develop our results. Let $A=\left(a_{i j}\right)$ be an $n \times n$ sign pattern matrix. By the directed graph $D(A)$ of $A$, we mean the directed graph with vertex set $\{1,2, \cdots, n\}$ and arc $(i, j)$ if $a_{i j} \neq 0$. A simple $k$-cycle $\gamma$ of length $|\gamma|=k$ of $A$ is a sequence of $k$ arcs $\left(i_{1}, i_{2}\right),\left(i_{2}, i_{3}\right), \cdots,\left(i_{k}, i_{1}\right)$ in $D(A)$ such that the vertices $i_{1}, i_{2}, \cdots, i_{k}$ are distinct. We denote the cycle $\gamma$ by $\left(i_{1}, i_{2}, \cdots, i_{k}, i_{1}\right)$. Write $\Pi_{\gamma}=a_{i_{1} i_{2}} a_{i_{2} i_{3}} \cdots a_{i_{k} i_{1}}$, the cycle product of. $A$ associated with a simple $k$-cycle $\gamma$. A simple $k$-cycle is said to be positive (respectively, negative) if it contains an even (respectively, odd) number of negative entries in its cycle product.

It is well-known that the determinant of a complex matrix $B$ is the sum of all possible terms of the form

$$
(-1)^{\left|\gamma_{1}\right|-1} \Pi_{\gamma_{1}}(-1)^{\left|\gamma_{2}\right|-1} \Pi_{\gamma_{2}} \cdots(-1)^{\left|\gamma_{p}\right|-1} \Pi_{\gamma_{p}}
$$

where $\gamma_{1}, \gamma_{2}, \cdots, \gamma_{p}$ are disjoint simple cycles the sum of whose lengths is equal to $n$. Similarly, $E_{k}(B)$, the sum of all $k \times k$ principlal minors of $B$, is equal to the sum of all terms of the form (1) where $\gamma_{1}, \gamma_{2}, \cdots, \gamma_{p}$ are disjoint simple cycles whose length sum

Received 27th April, 1995

Copyright Clearance Centre, Inc. Serial-fee code: 0004-9729/96 \$A2.00+0.00. 
equals $k$. The computation of the characteristic polynomial of $B$ is then expressed in terms of its cycle products as follows:

$$
P_{B}(t)=t^{n}+\sum_{k=1}^{n}(-1)^{k} E_{k}(B) t^{n-k}
$$

An $n \times n$ sign pattern matrix $A$ is said to be potentially nilpotent if it allows a nilpotent matrix, that is, there exists $B \in Q(A)$ such that $B$ is nilpotent. From (2), this is equivalent to finding $B \in Q(A)$ such that $E_{k}(B)=0$ for all $k$. We discuss potential nilpotence of star sign pattern matrices in section 2 , and find all possible potentially nilpotent $7 \times 7$ linear tree sign patterns in section 3 .

\section{Star Sign Patterns}

A directed graph on $n$ vertices is a star if it consists of $n-1$ simple 2-cycles which contain a common vertex $v$. The vertex $v$ is called the centre of the star graph. A star graph may permit loops. A sign pattern matrix $A$ is star if $D(A)$ is a star.

TheOREM 1. Let $A$ be an $n \times n$ star sign pattern matrix. If $D(A)$ has exactly one loop then $A$ is not potentially nilpotent.

Proof: If $D(A)$ has exactly one loop then for every $B \in Q(A), E_{1}(B) \neq 0$.

THEOREM 2. Let $A$ be an $n \times n$ star sign pattern matrix such that $D(A)$ has no loops. Then $A$ is potentially nilpotent if and only if $A$ has a positive and a negative simple 2-cycle.

Proof: Let $B \in Q(A)$. Then, from (2), $P_{B}(t)=t^{n}+E_{2}(B) t^{n-2}$, and

$$
E_{2}(B)=-\left(\Pi_{\gamma_{1}}+\Pi_{\gamma_{2}}+\cdots+\Pi_{\gamma_{n-1}}\right)
$$

where $\gamma_{1}, \gamma_{2}, \cdots, \gamma_{n-1}$ are the $n-1$ simple 2-cycles of $B$. Hence $E_{2}(B)=0$ if and only if there are terms in (3) of opposite sign.

Let $A$ be a star sign pattern matrix centred at $v$. Assume $D(A)$ consists of $k$ loops $v_{1}, v_{2}, \cdots, v_{k}$. Note that $v$ may or may not be one of the $k$ loops. Let $A_{1}$ be the sign pattern submatrix that lies in the rows and columns of $A$ indexed by $v, v_{1}, v_{2}, \cdots, v_{k}$; and let $A_{2}$ be the submatrix of $A$ obtained by deleting the rows and columns indexed by $\left\{v_{1}, v_{2}, \cdots, v_{k}\right\}-\{v\}$ and replacing $a_{v v}=0$.

Theorem 3. Let $A$ be an $n \times n$ star sign pattern matrix. Then $A$ is potentially nilpotent if and only if $A_{1}$ and $A_{2}$ are potentially nilpotent. 
Proof: Assume $v_{1}, v_{2}, \cdots, v_{k}$ are loops of $D(A)$ and $v_{k+1}, v_{k+2}, \cdots, v_{n}$ are nonloops of $D(A)$. Let $B \in Q(A)$. If the centre $v$ is a loop, say $v=v_{1}$, then

$$
E_{1}(B)=b_{v_{1} v_{1}}+b_{v_{2} v_{2}}+\cdots+b_{v_{k} v_{k}}
$$

$$
\begin{aligned}
E_{m}(B)=-\left(\alpha+\beta_{m}\right) b_{i_{1} i_{1}} b_{i_{2} i_{2}} & \cdots b_{i_{m-2} i_{m-2}}+b_{j_{1} j_{1}} b_{j_{2} j_{2}} \cdots b_{j_{m} j_{m}}, m=2,3, \cdots, k \\
E_{k+1}(B) & =\alpha b_{v_{3} v_{2}} b_{v_{3} v_{3}} \cdots b_{v_{k} v_{k}} \\
E_{m}(B) & =0, m=k+2, k+3, \cdots, n,
\end{aligned}
$$

where $\alpha=-\sum \Pi_{\gamma}$, and $\gamma$ runs over the simple 2-cycles $\left(v, v_{j}, v\right), j=k+1, k+2, \cdots, n$, and the vertices $i_{1}, i_{2}, \cdots, i_{m-2}$ run over the disjoint vertices from $\left\{v_{1}, v_{2}, \cdots, v_{k}\right\}$, and $\beta_{m}$ is the sum over all simple 2 -cycles, disjoint from $i_{1}, i_{2}, \cdots, i_{m-2}$, in $A_{1}$, and $j_{1}, j_{2}, \cdots, j_{m}$ are disjoint vertices from $\left\{v_{1}, v_{2}, \cdots, v_{k}\right\}$. If $A$ is potentially nilpotent then there exists $B \in Q(A)$ such that $E_{1}(B)=E_{2}(B)=\cdots=E_{k+1}(B)=0$. From (6), we have $\alpha=0$, and thus, by Theorem $1, A_{2}$ is potentially nilpotent. But then (5) becomes

$$
E_{m}(B)=-\beta_{m} b_{i_{1} i_{1}} b_{i_{2} i_{2}} \cdots b_{i_{m-2} i_{m-2}}+b_{j_{1} j_{1}} b_{j_{2} j_{2}} \cdots b_{j_{m} j_{m}}
$$

for $m=2,3, \cdots, k$. Hence from (4) and (7), $A_{1}$ is potentially nilpotent, and vice versa. A similar argument holds if the centre $v$ is not a loop.

Note that $A_{1}$ is a star sign pattern matrix which has loops at each vertex except possibly at the centre, and $A_{2}$ is a star sign pattern matrix without loops. According to Theorem 2 and Theorem 3 , it suffices to consider star sign pattern matrices which have loops at each vertex except possibly the centre vertex.

Theorem 4. Let $A$ be an $n \times n$ star sign pattern matrix such that each vertex has a loop except possibly at the centre. Then $A$ is potentially nilpotent if and only if:

(i) for $n=2$, the diagonals of $A$ have opposite signs and the simple 2-cycle of $A$ is negative;

(ii) for $n=3$, if the centre is not a loop, two diagonals of $A$ have opposite signs and $A$ has only negative simple 2-cycles;

(ii) for $n=3$, if the centre is a loop, the diagonals have positive and negative signs, the two simple 2-cycles have opposite signs if the noncentre vertices have the same sign; and it has only negative simple 2-cycles if the noncentre vertices have opposite signs.

Proof: Let $n=2$. By Theorem 1, $D(A)$ has two loops. The existence of $B \in Q(A)$ such that $P_{B}(t)=t^{2}-\left(b_{11}+b_{22}\right) t+\left(b_{11} b_{22}-b_{12} b_{21}\right)=t^{2}$, is equivalent to the stated conditions. 
Suppose $n=3$ and the centre is not a loop. Then for $B \in Q(A)$ we have

$$
\begin{aligned}
& E_{1}(B)=b_{i i}+b_{j j}, \\
& E_{2}(B)=b_{i i} b_{j j}-\Pi_{\gamma_{1}}-\Pi_{\gamma_{2}}, \\
& E_{3}(B)=-b_{i i} \Pi_{\gamma_{2}}-b_{j j} \Pi_{\gamma_{1}},
\end{aligned}
$$

where $i$ and $j$ are vertices having loops that are not in the simple 2-cycles $\gamma_{1}$ and $\gamma_{2}$ respectively. If $B$ is nilpotent then, by (8), $b_{i i}=-b_{j j}$. Substituting into (10), we have $\Pi_{\gamma_{1}}=\Pi_{\gamma_{2}}$, and then by (9), $\Pi_{\gamma_{1}}$ is positive.

Now suppose $n=3$ and the centre is a loop. Assume the centre is the vertex 1 . Let $B \in Q(A)$, and $u=b_{11}, v=b_{22}, w=b_{33}, x=b_{12} b_{21}$ and $y=b_{13} b_{31}$. Then $B$ is nilpotent if there exist nonzero real numbers $u, v, w, x, y$ such that

$$
\begin{gathered}
u+v+w=0 \\
-x-y+u v+u w+v w=0 \\
-w x-v y+u v w=0
\end{gathered}
$$

If $v=w$ then dividing (13) by $v$ and substracting from (12), we have

$$
v(u+w)=0
$$

Substituting (11) into (14) we obtain $v^{2}=0$, a contradiction. Hence $v-w \neq 0$. Solving the equations (12) and (13) for $x$ and $y$, we have

$$
x=-\frac{v^{3}}{v-w} \text { and } y=\frac{w^{3}}{v-w} .
$$

From (11), the sign and the value of $u$ are determined by $v$ and $w$. There are four possible signs for $v$ and $w$. Using (15) we see that if $\operatorname{sign}(v, w)=(+,+)$ or $(-,-)$ then $\operatorname{sign}(x, y)=(+,-)$ or $(-,+)$, and if $\operatorname{sign}(v, w)=(+,-)$ or $(-,+)$ then $\operatorname{sign}(x, y)=$ $(-,-)$. This proves (iii).

The method used in (iii) may be applied to the case when $n \geqslant 4$. Of course, for large values of $n$, the computations by Cramer's rule for the non-homogeneous systems are fairly involved.

\section{Linear TREe Sign Patterns}

A sign pattern matrix $A=\left(a_{i j}\right)$ is tree sign pattern (t.s.p.) [4] if

(i) $D(A)$ is strongly connected without loops;

(ii) there is no simple cycle of length 3 or more;

(iii) $a_{i j} \neq 0$ if and only if $a_{j i} \neq 0$. 
A tree is linear if no vertex is adjacent to 3 or more vertices. It looks like:

$$
1 \rightleftarrows 2 \rightleftarrows \cdots \rightleftarrows n
$$

It is clear that every linear tree sign pattern matrix is permutationally similar to a tridiagonal sign pattern matrix with zero main diagonal. Hence we may asssume without loss of generality that a linear t.s.p. matrix is tridiagonal with zero main diagonal. For convenience, a tridiagonal matrix $B=\left(b_{i j}\right) \in Q(A)$ with zero main diagonal is sometimes expressed as

$$
B=\operatorname{tridiag}\left(\left(b_{12}, b_{23}, \cdots, b_{n-1}\right),\left(b_{21} b_{32}, \cdots, b_{n n-1}\right)\right)
$$

Suppose $A$ is a linear t.s.p. matrix of even order. Then it is clear that $A$ is not potentially nilpotent since the composite $n$-cycle of $B \in Q(A)$ is nonzero, and thus has a nonzeo determinant. Therefore, in the following, we discuss only odd order linear t.s.p. Let $A$ be a tridiagonal linear t.s.p. matrix of order $n$. The arc pattern of $A$ is the $(n-1)$-tuple $\left(a_{1}, a_{2}, \cdots, a_{n-1}\right)$, where $a_{i}=a_{i} i_{1+1} a_{i+1} i, i=1,2, \cdots, n-1$. From [1], $A$ is potentially nilpotent if and only if its arc pattern is $\pm(+,-)$ when $n=3$; and that its arc pattern is one of $\pm(+,+,-,+), \pm(+,-,+,+)$ and $\pm(+,-,+,-)$ when $n=5$. In the following, we determine the case when $n=7$.

Let $A$ be an $7 \times 7$ linear t.s.p. matrix. We assume that $A$ is tridiagonal with zero main diagonal. For each $B=\left(b_{i j}\right) \in Q(A)$, let $b_{i}=b_{i+1} b_{i+1} i, i=1,2, \cdots, n-1$. From (2), $B$ is nilpotent if and only if

$$
\begin{aligned}
& b_{1}+b_{2}+b_{3}+b_{4}+b_{5}+b_{6}=0 \\
& b_{1}\left(b_{3}+b_{4}+b_{5}+b_{6}\right)+b_{2}\left(b_{4}+b_{5}+b_{6}\right)+b_{3}\left(b_{5}+b_{6}\right)+b_{4} b_{6}=0 \\
& b_{1} b_{3}\left(b_{5}+b_{6}\right)+b_{4} b_{6}\left(b_{1}+b_{2}\right)=0
\end{aligned}
$$

We have $2^{6}=64$ possibilities to specify the signs of the arc pattern of $A$. But since $\left(b_{1}, b_{2}, b_{3}, b_{4}, b_{5}, b_{6}\right)$ is a solution of the system (16)-(18) if and only if $\left(-b_{1},-b_{2},-b_{3},-b_{4}\right.$, $\left.-b_{5},-b_{6}\right)$ is a solution of the system, we reduce the possible arc patterns to 32 patterns with $b_{1}$ being positive. Without loss of generality, we may assume that $b_{1}=1$. From (18) we have

$$
b_{3}\left(b_{5}+b_{6}\right)+b_{4} b_{6}=-b_{2} b_{4} b_{6}
$$

Substituting (16) and (19) into (17), we obtain

$$
\left(-b_{1}-b_{2}\right)+b_{2}\left(b_{4}+b_{5}+b_{6}\right)-b_{2} b_{4} b_{6}=0
$$


Direct computations from (20), give that

$$
\left(b_{2}+1\right)^{2} / b_{2}=-b_{3}-b_{4} b_{6} .
$$

Set $p=\left(b_{2}+1\right)^{2} / b_{2}$ and $q=b_{4} b_{6}$. Then (21) becomes

$$
b_{3}=-(p+q)
$$

and (19) becomes

$$
b_{5}+b_{6}=\left(1+b_{2}\right) q /(p+q) .
$$

From (22), (23), and (16) we have

$$
\begin{aligned}
b_{4} & =-1-b_{2}-b_{3}-b_{4}-b_{5}-b_{6} \\
& =-\left(1+b_{2}\right)+(p+q)-\left(1+b_{2}\right) q /(p+q) \\
& =\Delta /(p+q),
\end{aligned}
$$

where

$$
\Delta=(p+q)^{2}-(p+q)\left(1+b_{2}\right)-q\left(1+b_{2}\right) .
$$

From (24)

$$
b_{6}=q / b_{4}=q(p+q) / \Delta,
$$

and then from (23) and (26),

(27) $b_{5}=q\left(1+b_{2}\right) /(p+q)-b_{6}=q\left(1+b_{2}\right) /(p+q)-q(p+q) / \Delta=q^{3} b_{2} /(\Delta(p+q))$.

Now we are ready to discuss the 32 arc patterns of $A$.

$p_{1}=(+,+,+,+,+,+)$ : Clearly $A$ is not potentially nilpotent.

$p_{2}=(+,+,+,+,+,-)$ : This pattern implies that $p>0$ and $q<0$. From (22), $p+q=-b_{3}<0$, and by (25), $\Delta>0$. Then we obtain, from (24), $b_{4}<0$, a contradiction.

$p_{3}=(+,+,+,+,-,+):$ We have $p>0$ and $q>0$, and thus, by (22), $b_{3}<0$, a contradiction.

$p_{4}=(+,+,+,+,-,-):$ We have $p>0$ and $q<0$. By (22), $p+q<0$. Then from (23), $b_{5}+b_{6}>0$, a contradiction.

$p_{5}=(+,+,+,-,+,+):$ Let $B \in Q(A)$ be defined by

$$
B=\operatorname{tridiag}\left((1,1,1,1,1,1),\left(1,1,2,10, \frac{27}{5}, \frac{3}{5}\right)\right) .
$$


Then $B$ satisfies equations (16)-(18), and thus is a nilpotent matrix.

$p_{6}=(+,+,+,-,+,-):$ We have $p>0$ and $q>0$. Then by $(22), b_{3}=-(p+q)<$ 0 , a contradiction.

$p_{7}=(+,+,+,-,-,+):$ We have $p>0, q<0$ and $p+q<0$, and by (25), $\Delta>0$, and thus from (27), $b_{5}>0$, a contradiction.

$p_{8}=(+,+,+,-,-,-):$ We have $p>0$ and $q>0$, and thus, by (22), $b_{3}<0$, a contradiction.

$p_{9}=(+,+,-,+,+,+)$ : This is a permutation similar to $p_{5}$.

$p_{10}=(+,+,-,+,+,-):$ We have $p>0$ and $q<0$. From (22), $p+q>0$. By (24), $\Delta>0$, and then, by (27), $b_{5}<0$, a contradiction.

$p_{11}=(+,+,-,+,-,+):$ We have $p>0$ and $q>0$, and from (22), $p+q>0$. Again by (24), $\Delta>0$, and then, by (27), $b_{5}>0$, a contradiction.

$p_{12}=(+,+,-,+,-,-):$ We define $B \in Q(A)$ as follows:

$$
B=\operatorname{tridiag}((1,1,1,1,1,1),(1,1,-2,2,-1,-1)) .
$$

Then $B$ satisfies equations (16)-(18), and thus is a nilpotent matrix.

$p_{13}=(+,+,-,-,+,+):$ We have $p>0, q<0$ and $p+q>0$. Then, by (23), $b_{5}+b_{6}<0$, a contradiction.

$p_{14}=(+,+,-,-,+,-):$ We have $p>0, q>0$ and $p+q>0$. Then by (24), $\Delta<0$, and thus by (27), $b_{5}<0$, a contradiction.

$p_{15}=(+,+,-,-,-,+)$ : We have $p>0, q<0$ and $p+q>0$. From (24) we have, $\Delta<0$, and thus by (27), $b_{5}>0$, a contradiction.

$p_{16}=(+,+,-,-,-,-):$ We have $p>0, q>0$ and $p+q>0$. Then, from (23), $b_{5}+b_{6}>0$, a contradiction.

$p_{17}=(+,-,+,+,+,+)$ : This is a permutation similar to $p_{3}$.

$p_{18}=(+,-,+,+,+,-):$ Let $B \in Q(A)$ be defined by

$$
B=\operatorname{tridiag}\left((1,1,1,1,1,1),\left(1,-2,1, \frac{1}{2}, \frac{1}{2},-1\right)\right) .
$$

Then $B$ satisfies equations (16)-(18), and thus is a nilpotent matrix.

$p_{19}=(+,-,+,+,-,+):$ Define a matrix $B \in Q(A)$ by

$$
B=\operatorname{tridiag}\left((1,1,1,1,1,1),\left(1,-\frac{1}{2}, \frac{1}{6}, \frac{1}{3},-2,1\right)\right) .
$$

Then $B$ satisfies equations (16)-(18), and thus is a nilpotent matrix.

$p_{20}=(+,-,+,+,-,-):-p_{20}$ is a permutation similar to $p_{14}$.

$p_{21}=(+,-,+,-,+,+)$ : This is a permutation similar to $p_{11}$. 
$p_{22}=(+,-,+,-,+,-):$ Let $B \in Q(A)$ be defined by

$$
B=\operatorname{tridiag}\left((1,1,1,1,1,1),\left(1,-\frac{3}{2}, \frac{1}{12},-\frac{1}{12}, \frac{3}{2},-1\right)\right) \text {. }
$$

Then $B$ satisfies equations (16)-(18), and thus is a nilpotent matrix.

$p_{23}=(+,-,+,-,-,+):$ Define $B \in Q(A)$ by

$$
B=\operatorname{tridiag}\left((1,1,1,1,1,1),\left(1, \frac{-1}{2}, \frac{3}{2}, \frac{-7}{3}, \frac{2}{21}, \frac{3}{7}\right)\right) \text {. }
$$

Then $B$ satisfies equations (16)-(18), and thus is a nilpotent matrix.

$p_{24}=(+,-,+,-,-,-):-p_{24}$ is a permutation similar to $p_{6}$.

$p_{25}=(+,-,-,+,+,+)$ : This is a permutation similar to $p_{7}$.

$p_{26}=(+,-,-,+,+,-):$ We have $p \leqslant 0$ and $q<0$. Then, from (22), $b_{3} \geqslant 0$, a contradiction.

$p_{27}=(+,-,-,+,-,+):$ This is a permutation similar to $p_{23}$.

$p_{28}=(+,-,-,+,-,-):-p_{28}$ is a permutation similar to $p_{10}$.

$p_{29}=(+,-,-,-,+,+):$ This is a permutation similar to $p_{15}$.

$p_{30}=(+,-,-,-,+,-):-p_{30}$ is a permutation similar to $p_{18}$.

$p_{31}=(+,-,-,-,-,+):$ We have $p \leqslant 0$ and $q<0$. Then, by (22), $b_{3} \geqslant 0$, a contradiction.

$p_{32}=(+,-,-,-,-,-):-p_{32}$ is a permutation similar to $p_{2}$.

Summarising, we have

Theorem 5. Let $A$ be an $7 \times 7$ tridiagonal linear t.s.p. matrix. Then $A$ is potentially nilpotent if and only if the arc pattern of $A$ is one of the forms

$$
\pm p_{5}, \pm p_{8}, \pm p_{12}, \pm p_{18}, \pm p_{19}, \pm p_{22}, \pm p_{23}, \pm p_{27}, \pm p_{30}
$$

Note that $p_{5}$ and $p_{9}$ are permutation similar, and so are $p_{23}$ and $p_{27}$.

\section{REFERENCES}

[1] S.C. Chang, 'On potentially nilpotent sign patterns', (preprint).

[2] C.A. Eschenbach and C.R. Johnson, 'Several open problems in qualitative matrix theory involving eigenvalue distribution', Linear and Multilinear Algebra 24 (1988), 79-80.

[3] C.A. Eschenbach and C.R. Johnson, 'Sign patterns that require real, nonreal or pure imaginary eigenvalues', Linear and Multilinear Algebra 29 (1991), 299-311.

[4] C.R. Johnson and C. Jeffries, 'Some sign patterns that preclude matrix stability', SIAM J. Matrix Anal. Appl. 9 (1988), 19-25. 